Products and Services In Practice is provided to readers using text and images from the manufacturer, supplier or distributor and does not imply endorsement by $B D J$ In Practice. Normal and prudent research should be exercised before purchase or use of any product mentioned.

Please send product and services news through to David Westgarth, BDJ In Practice via: David.Westgarth@bda.org

\section{Achieving artful precision every time}

A truly professional clean is a fine art, requiring a careful combination of precise engineering and in-depth clean. Enter the Hydrosonic Pro toothbrush by Curaprox - delivering a professional clean feeling every time.

Engineered with innovative

CURACURVE bend technology, the Hydrosonic Pro brush head effortlessly twists and turns to reach those hard-toreach spots, making it the perfect partner for patients with orthodontic appliances, implants, or sensitive gums.

Exceptional performance comes as standard as ultrasound technology provides a dual cleaning function. This means patients can choose between mechanical and hydrodynamical settings, while CUREN bristles further enhance the experience with a compact cleaning surface.

Grant your patients unparalleled performance and precision today with the easy-to-handle Hydrosonic Pro toothbrush by Curaprox.

For more information call 01480 862084, email info@curaprox.co.uk or visit www.curaprox.co.uk.

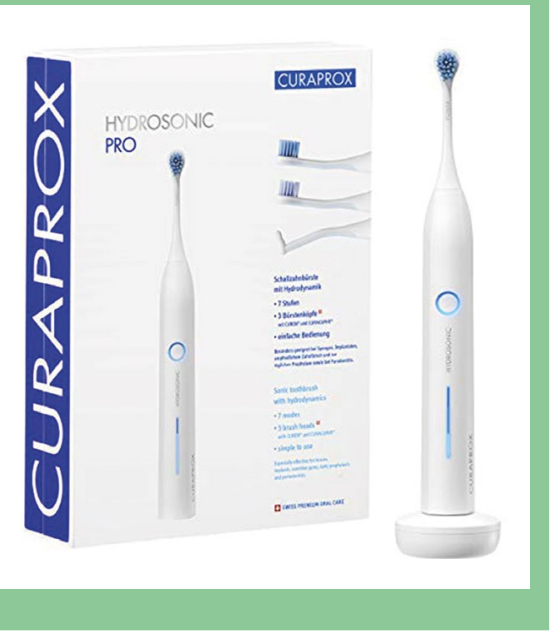

\title{
Reimagine your orthodontic workflows
}

With orthodontic aligners becoming such a popular choice for patients, it makes sense to invest in an innovative solution that streamlines your orthodontic workflows from start to finish. The new CS Model+ v5 software from Carestream dental is your key to excellence in aligners.

With this cutting-edge software you can design and create aligners faster than ever before. Evaluate digital models, create virtual orthodontic setups and develop an aligner plan in minutes - with CS Model+ v5 everything is simple and stress-free giving you the opportunity to provide exceptional outcomes.

Plus, as the software uses state-of-the-art AI technology, you can guarantee that it will expand your orthodontic treatment capabilities and allow you to initiate quick, reliable treatment for those who need treatment in a shorter timeframe.

For more information contact Carestream Dental on 08001699692 or visit www.carestreamdental.co.uk.

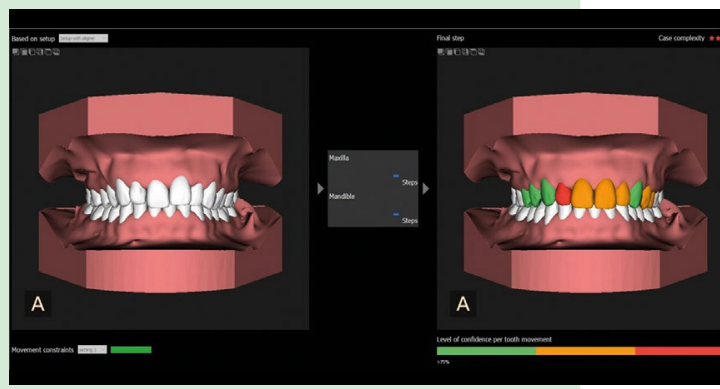

\section{Effective cleaning and sterilisation of dental handpieces}

In immediate response to the pandemic last summer, dental equipment manufacturers acted swiftly, adapting their products to minimise aerosol generation and providing practitioners with alternative non-AGP treatment options. For example, NSK's Ti-Max Z95L and Z45L handpieces now have an anti-suck back mechanism that prevents blood and foreign matter entering the head, and a switch to turn off the air/ water mix so that only water spray is used, thereby minimising aerosols.

But cleaning the hidden internal surfaces and lumens of a handpiece remains a challenge. This essential cleaning is difficult to achieve using a manual process which can be inefficient and is difficult to reproduce consistently to a validated standard, potentially resulting in nonsterile instruments and even damage to the autoclave.

The ideal method of internal cleaning and lubrication is to use an automated unit, such as the NSK iCare. This equipment features technology specifically designed to quickly and effectively remove dirt and debris from the internal workings of handpieces and deliver the precise amount of lubricant in preparation for sterilisation.

One of the most complex challenges faced by decon teams is how to ensure effective sterilisation of their handpieces. NSK's iMax S and iClave plus vacuum autoclaves have been developed to sterilise all instruments, especially dental handpieces. The utilisation of a thermally efficient copper chamber results in improved performance, offering faster, more efficient cycles compared with traditional stainless steel chambered autoclaves of an equivalent size and capacity.

Find out more about the NSK SMART3 decontamination protocol and how you can protect your handpieces at mynsk.co.uk/ decontamination/. 